\title{
Vibratory behaviour of glass fibre reinforced polymer (GFRP) interleaved with Nylon Nanofibers
}

\author{
Cristobal Garcia ${ }^{a *}$, Jodi Wilson ${ }^{a}$, Irina Trendafilova ${ }^{a}$, Liu Yang ${ }^{a}$ \\ ${ }^{a}$ University of Strathclyde, Mechanical and Aerospace Department, 75 Montrose Street, G1 \\ 1XJ, Glasgow, UK
}

Email: cristobal.garcia@strath.ac.uk

\begin{abstract}
The main purpose of this study is to investigate the influence of the inclusion of nylon nanofibers on the global dynamic behaviour of GFRP composite laminates. The vibration behaviour of GFRP composites reinforced with nylon nano-fibres is considered experimentally and numerically using a finite element model. The present analysis of clamped-clamped beams investigates the natural frequencies, the damping and the stiffness of virgin and nano-interleaved composite laminates. The numerical modelling uses ANSYS Workbench 16.2. Experimental and numerical results showed a significant effect of the nylon nanofibers on the dynamic behaviour of the composites. Nano-modified composites demonstrated a consistent increase in the damping ratio and inter-laminar strength. However, the variation in natural frequencies and stiffness due to the nanofibers was very small. This study contributes to the knowledge about the macro dynamic properties of nylon interleaved GFRP composites. It demonstrates that a simple FE model can be used to accurately predict the dynamic behaviour of such nano-composites.
\end{abstract}

Keywords: Composite; Vibration; Natural Frequency; Damping Ratio; Stiffness; FEM.

\section{Introduction}

Composite laminates reinforced with nanofibers are becoming more and more important in a wide range of sectors such as aerospace, automotive and marine engineering because of their outstanding properties. The comparison between standard composite materials and composites reinforced with nanofibers shows that the inclusion of nanofibers in composites can drastically improve some materials properties. Thus many authors investigated how the inclusion of nanofibers in composites can enhance some of their properties [1-5].

Delamination is a particularly dangerous failure mode for laminates because it takes place and grows under the surface without being visible from outside. Delaminated composites can lose up to $60 \%$ of its strength and stiffness because of delamination and still remain 
unchanged. It is known that the inclusion of nanofibers into the ply-to-ply interfaces has the ability to prevent delamination [1-2]. Some nano-enrichments can make composites more resistant to impacts, plastic deformation and fracture than standard composites: It has been observed that the inclusion of nanofibers in composites is one of the ways to enhance the mechanical properties in composites. Thus, many authors have extensively investigated how the inclusions of nanofibers into composites increase the resistance to impact as compared to virgin composites [5-6].

Composites reinforced with piezoelectric nanofibers have potential applications in selfsensing materials [7-9]. Nanofibers with piezoelectric behaviour can be homogenously dispersed into the ply-to-ply interfaces of composites with the aim of monitoring the health state of the composite. The piezoelectric nanofibers can convert mechanical energy into electrical energy and vice-versa. Therefore, if mechanical energy is applied to the nanoreinforced composite through impact, the piezoelectric nanofibers will output an electrical signal. This electrical signal can provide information on the magnitude of the impact and its location enabling the capability of self-sensing. Composites reinforced with core-shell nanofibers can be even used to heal the composite in case of local damage [10-12]. The development of such self-healing composites is typically carried out by the incorporation of two types of core-shell nanofibers into the ply interfaces of the composite. These two types of core-shell nanofibers are in contact with each other. The first type of core-shell nanofibers is filled with a healing agent and the other is filled with a hardener. In case of local damage, both of the core-shell nanofibers are broken and release healing agent and hardener ready to flow to the damaged area. As a result, the damaged area can be repaired by new resin created through the reaction between the healing agent and hardener.

There is still very little research on the effect of nanofibers on the dynamic and vibratory properties of structures made of nano-enriched composites. On the other hand, most structures are subjected to vibration including aircrafts, vehicles and buildings. It is widely known that vibrations are responsible for an considerable number of accidents in aircrafts, nuclear plants, bridges and other civil engineering structures that fail (are destroyed) as a results of a large amplitude vibrations. Consequently, there is a need for reduction of vibration on structures made of composite materials. One of the methods of supressing vibration is to increase the damping, which can be achieved by incorporating nanofibers. Furthermore the knowledge about the macro-dynamic behaviour of the nano-enriched composite is a pre-requisite for the development of nano-included dynamic/vibratory sensors.

There are a few attempts to model the mechanical and dynamic macro behaviour of nano sized material structures and nano enriched materials using different finite elements. A number of these are devoted to carbon nanotubes. Special attention deserves the works of Ribeiro [13] which attempts to develop a specific finite element model to model the vibration behaviour and to analyse internal resonances of beams with dimensions of a few 
nanometers. This study uses Bernouli -Euler $p$-version beam elements to model the behaviour of nano-sized beams. It also reports a comparison with published experimental results on cabon nano-tubes. The works of Rafiee and Moghadam [14] which suggest finite element approach for modal behaviour of nano-tube reinforced polymers should also be mentioned. They use finite element model for the purpose and perform modal analysis for two types of boundary conditions. Their results show considerable growth of the natural frequencies as compared to the virgin polymer. The same authors consider post impact behaviour of carbon nano-tubes enriched polymer using a multi-scale finite element model [15] and their results show improvement of the impact resistance of the carbon nanoenriched polymer as compared to the virgin/pure one. There is just one study which considers the macro-mechanical behaviour of nylon fibres, which is a purely experimental one and concentrates on its visco-elastic behaviour when subjected to large sinusoidal stress changes [16]. This study confirms the nonlinear behaviour /response of nylon fibres when subjected to harmonic stress loading but the loading applied is quasi static and the observed behaviour is long term steady-state deformation. The present investigation attempts to analyse the free dynamic response of nylon enriched composite laminates. It is based on a simple FEM and on a specifically developed experimental programme to analyse the free decay response of virgin and nano-enriched composites. The study proves and demonstrates that a simple FE model can give accurate enough prediction of the vibratory behaviour of nano-enriched laminates. It also makes new conclusions on the influence of nylon nano layers on the macro dynamic behaviour and properties of the composite laminates.

Although some authors have studied the dynamic behaviour of nano-enriched composites mainly based on experimental method [17-18], there is currently very little information on the effect of nylon nano-fibrous enrichment and its effect on the global/macro dynamical properties of the enriched structures. Some of the authors of this study previously investigated some effects of the inclusion of nylon layers in carbon-fibre composites [17]. [17] is a completely experimental study and the authors concentrated on the effect of the nano-layers when they are interleaved in different places. It was focussed primarily on the energy dissipation of the nano-enriched specimens and their resistance to impact. It has to be noted that nanofibers of nylon are frequently used to reinforce composites since nylon exhibits high melting temperature allowing the nanofibers to maintain their morphology during the curing process of the laminate at approximately $120^{\circ} \mathrm{C}$. Nylon has excellent mechanical properties compared to other polymers used to electrospun. The cost of the nylon and its solvents is reasonable in comparison to others polymers/solvents. The viscosity of the solution of nylon is low enough to electrospun the nanofibers. Nylon shows good adhesion and compatibility to be bonded with epoxy.

This study aims to contribute and to further the knowledge and the understanding of the properties and the dynamic behaviour of nylon nano-enrichment for glass-fiber epoxy composites. It investigates the influence of nylon nanofibers on dynamic properties of glass 
fibre reinforced polymer composites. It develops a relatively simple finite element model based on non-linear (transient) vibratory analysis, which is capable to accurately predict the dynamic behaviour of nylon nano-interleaved GFRP composites. Historically, most structural vibration models are linear and they assume linearity of the structure and the material. Linear analysis can provide an acceptable approximation for most cases of homogeneous materials. However, composites and especially nano-enriched ones are characterized by a complex non-linear behaviour due to their heterogeneous nature. As a result, neglecting their inherent nonlinear behaviour can potentially lead to serious errors in the modelling and the estimation of their properties. A number of publications investigated the behaviour of composites and nano-enriched composites and concluded that they are characterized by well-expressed nonlinear behaviour [19-21]. This study also proves that using transient vibration analysis rather than modal analysis results in better/more precise prediction of the resultant vibratory behaviour. In this paper the dynamic behaviour of virgin and nylon interleaved GFRP composites is investigated experimentally and numerically (using the mentioned FE modelling) and the changes in the dynamic parameters due to the nanoenrichment are studied. The FE model was created mainly for the purposes mainly for the purposes of simple simulation of the free dynamic response of nano interleaved composites and also for the purposes of validating the experimental results. But by the comparison of the experimental and the numerical results we were also able to show that the FE model is in adequate agreement with the experimental results and thus can be used to accurately enough model and predict the free dynamic behaviour of nano-interleaved GFRP composites. It should be mentioned that this is the first attempt, although simple enough, to provide adequate modelling of the free vibration response of nylon interleaved GFRP composites. The authors are on the opinion that this model can be used for predicting the dynamic behaviour of other nano enriched composites and its simplicity makes it easy to implement. The experimental program was specifically developed for the purposes of testing the free decay response of the virgin and the nano interleaved specimens and then the numerical simulations are made to mimic closely the experiments and the tested specimens.

The paper is organised as follows. Section 2 explains how virgin and nano-enriched composites were fabricated. Section 3 indicates how these composites were tested experimentally. A finite element model of these composites is developed using ANSYS Workbench 16.2 is introduced in section 4. This section also explains how the model is refined and how the dynamical properties are simulated using both modal and transient analysis. Furthermore, the results obtained from the finite element model are compared with the experimental ones to validate the model. In \& 5 the effect of the nylon enrichment on the dynamic properties of GFRP composites is discussed on the basis of the experimental and the simulation results. Eventually some conclusions about the vibratory behaviour and macro-dynamic properties of nano interleaved GFRP composites are offered. 


\section{Virgin and Nano-composites fabrication.}

Composite laminates beams without nanofibers (known as virgin composite) were manufactured by hand lay-up of ten layers of unidirectional EC9 5X136 tex glass fibre/epoxy resin prepreg supplied by G.Angeloni Srl. The dimensions of the beams are $168 \times 32 \mathrm{~mm}$, the thickness is $3 \mathrm{~mm}$. The stacking sequence was $[0 / 90 / 0 / 90 / 0]_{s}$ for a total of 10 layers. The thickness of each layer of GFRP is $0.3 \mathrm{~mm}$. Composite laminates beams enriched with nylon nanofibers (known as nano composite) were also manufactured by hand lay-up with identical sizes and stacking sequence as the virgin (non-modified) ones. However, 8 layers of nylon nanofibers and epoxy resin were interleaved into the composite. The layers of nanofibers were located at each interface, excluding the central one. The sample beams were tested in clamped-clamped configuration, and the impact induces primarily flexuraltype of loads, which induce bending stresses and strains, and they become negligible in the mid-interface. Consequently, it was considered unnecessary to interleave the less-stressed middle interface. The thickness of each nano-layer of nylon was about $25 \mu \mathrm{m}$. The inclusion of nanofibers had a negligible effect on the thickness of the specimens. After the lay-up, the specimens were cured in autoclave at $150^{\circ} \mathrm{C}$ for one hour. The heating rate employed in the curing was $2^{\circ} \mathrm{C} / \mathrm{min}$ and the pressure was 6.4 bars.

Nylon nanofibers were manufactured by electrospinning technique. Nylon Zytel E53 NC010 in the form of pellets was used and formic acid and Chloroform were used as solvents. The first step in the preparation of the nanofibers was to dissolve the polymer nylon Zytel in a solution of Formic Acid and Chloroform (50:50 v/v) at a concentration of $14 \% \mathrm{w} / \mathrm{v}$. Secondly, the solution was transferred to an electrospinning apparatus for producing nanofibers. Finally, the electrospinning of nanofibers was carried out using the following conditions: applied voltage $23 \mathrm{kV}$, feed rate $0.3 \mathrm{~mL} / \mathrm{h}$ per needle, needle-tip to collector distance $10 \mathrm{~cm}$, room temperature, relative humidity of $40 \%$. The SEM image of electrospun nanofibers [24] is given in figure 1 (a) and the fiber diameter distribution is presented in figure 1 (b). The nanofibers were electrospun in random directions as can be seen from Figure 1 (a). The nanofibers diameter was measured on the basis of the SEM image using image-processing software Image J 1.45s. The nanofibers mean diameter of nylon was measured to be 200 $\mathrm{nm}$. The diameter has a heavy tailed distribution which is skewed to the right thus indicating that the diameter is somewhere between 150 and $250 \mathrm{~nm}$ with a probability of about $65 \%$ (see figure 1(b)).The fiber diameter distribution was determined by measuring 100 fibres. The nanofibers are kept under vacuum over Diphosphorus pentoxide at room temperature overnight to remove residual solvents before being hand-interleaved into the laminate during the lay-up. The final nanofibrous material had a surface density of $28.5 \mathrm{~g} / \mathrm{m} 2$. The procedure to evacuate air from the nanofibrous material which also enables a good resin infiltration into the nanofibrous material was vacuum treatment. The vacuum treatment to evacuate air was carried out during the curing. 
(a)

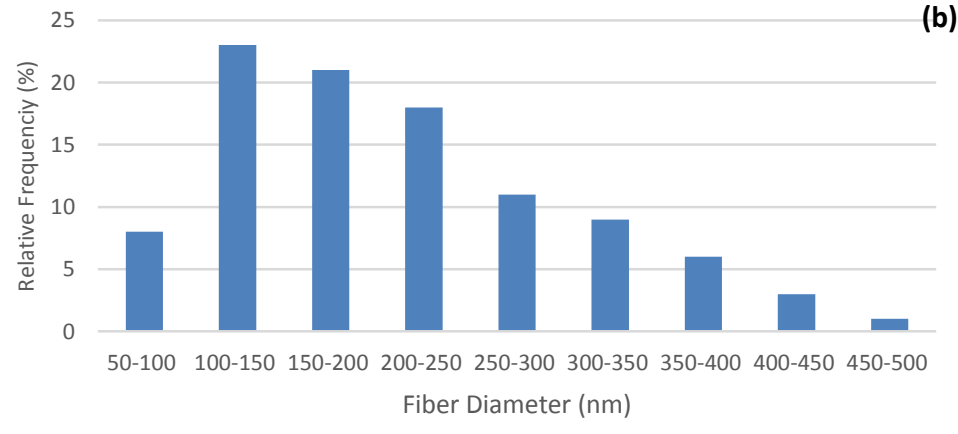

(b)

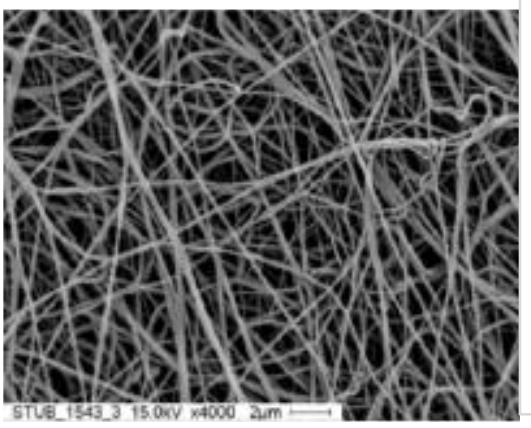

Figure 1: (a) SEM image of electrospun nylon nanofibers and (b) fiber diameter distribution of nylon nanofibers

\section{Vibration Testing}

The characterization of the dynamical behaviour for virgin and nano composites was carried out by means of free decay vibration tests. The free decay acceleration signals were used to calculate the natural frequencies and the damping ratio. The tests were carried out with the set-up shown in figure 2 . During the tests, the beams were clamped at both ends and an external excitation (sharp impact) was applied at the middle of the beam using a modal hammer. The beams were $168 \mathrm{~mm}$ long, $32 \mathrm{~mm}$ wide and $3 \mathrm{~mm}$ thick. The clamped regions were $8 \mathrm{~mm}$ long, thus the free span of the beams was $152 \mathrm{~mm}$. An accelerometer was attached at $60 \mathrm{~mm}$ from the left support and mounted on the top surface of the specimen to register the beams' responses, as shown in figure 2 . The accelerometer was strictly placed in the same position for all experiments. The mass of the accelerometer was $55 \mathrm{~g}$. The free decay acceleration signal of each specimen was recorded by a RT-440 portable analyser. During the vibration tests, the signals were recorded for $0.5 \mathrm{~s}$ and sampled at 5000 $\mathrm{Hz}$. The procedure was repeated 10 times to obtain a multiple number of realizations for each specimen. It should also be noted that all the measurements were taken on the same test rig to ensure the same boundary conditions for all tests.
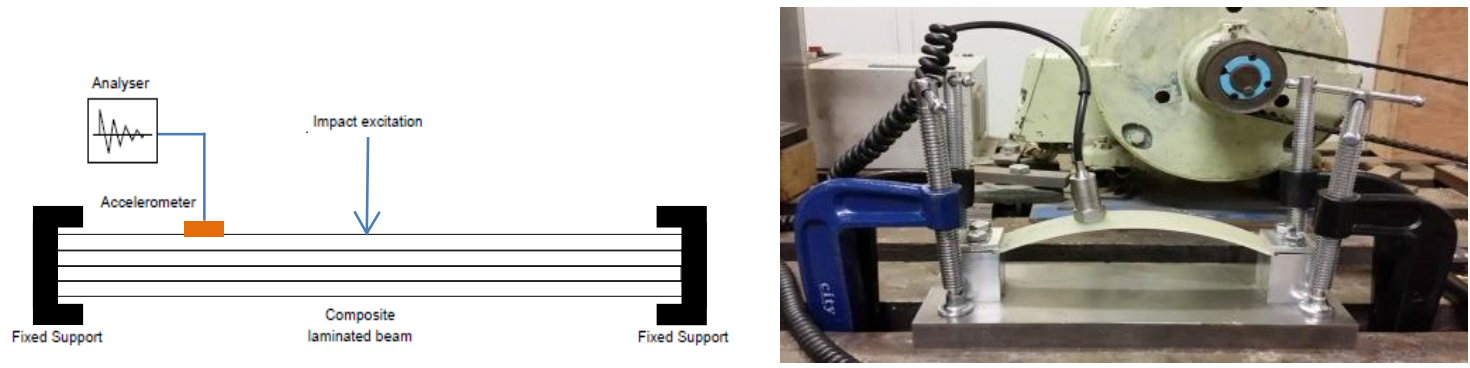

Figure 2: Dynamic free decay experiment for measuring the vibratory responses of the composite laminated beams. 
The first five natural frequencies of the sample beams were determined using the free decay acceleration signals in the frequency domain, as shown in figure 2 . The free decay time domain response is given in Figure $3(a)$ and Figure $3(b)$ represents the signal spectrum. The distinct peaks in the resulting spectrum were assumed to indicate the natural frequencies.

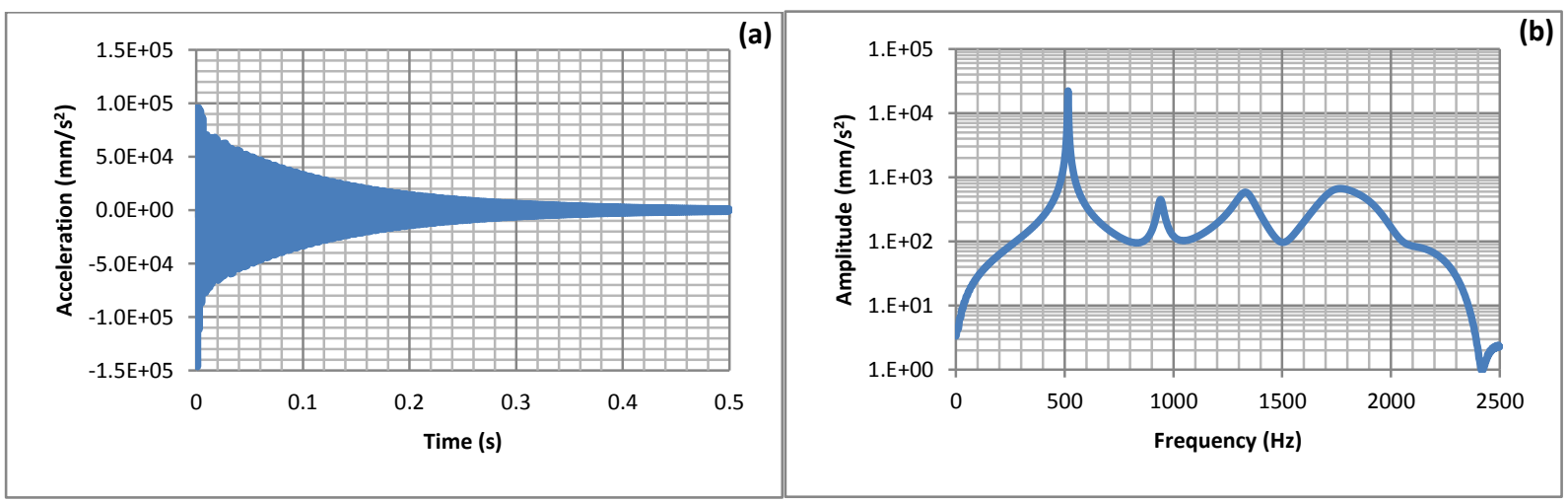

Figure 3: Vibratory response of virgin composite beam. (a) Free decay acceleration signal and (b) frequency spectrum of the free decay acceleration signal

The damping ratio was calculated using the time domain of the free decay acceleration signal by the Logarithmic Decrement method [22-23]. Two peaks of the free decay acceleration signal are used to calculate the logarithmic decrement $\delta$ using Eq. (1).

$$
\delta=\frac{1}{n} \ln \left(\frac{y_{0}}{y_{n}}\right)
$$

Where $y_{0}$ is the acceleration of the first peak and $y_{n}$ was the acceleration of the second peak, taken $n$ waves later. The damping ratio $\zeta$ was calculated from the logarithmic decrement using Eq. (2).

$$
\zeta=\frac{1}{\sqrt{1+\left(\frac{2 \pi}{\delta}\right)^{2}}}
$$

\section{Modelling the vibratory behaviour of the virgin and nano enriched specimens}

\subsection{Finite Element Modelling}

A finite element model was developed in ANSYS Workbench 16.2 to simulate the free decay behaviour of virgin and nano composites. The element type used in the finite element model was Shell 181. This is a four-noded element (I, J, K, and L) with six degrees of freedom 
$(1,2,3,4,5$, and 6$)$ at each node: translations in the $x, y$ and $z$ directions, and rotations about the $x, y$ and $z$ axes. The element thickness is assigned to be equal to that of the corresponding individual lamina. The finite element model is defined by elements and nodes forming a mesh.

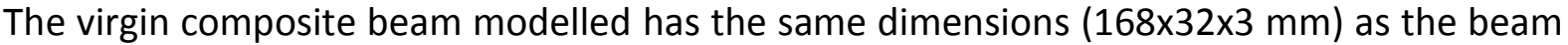
manufactured experimentally. The number of layers in the composite was ten unidirectional glass fibre/epoxy layers. The thickness of each layer was modelled as $0.3 \mathrm{~mm}$. The ply orientations of the layers were $[0 / 90 / 0 / 90 / 0]_{s}$ and the materials constants used are $E_{1}=44$ $\mathrm{GPa}, \mathrm{E}_{2}=\mathrm{E}_{3}=11 \mathrm{GPa}, \mathrm{G}_{12}=\mathrm{G}_{13}=4.5 \mathrm{GPa}, \mathrm{G}_{23}=0.5 \mathrm{GPa}, \mathrm{v}_{12}=0.28, \mathrm{v}_{13}=\mathrm{v}_{23}=0.1$ and $\rho$ $=1085.9 \mathrm{Kg} \mathrm{m}^{-3}[24]$.

The nano enriched composite beam modelled had the same dimensions, number of layers, material type and ply orientations as the virgin composite model however layers made of nylon nanofibers and epoxy resin were embedded into the ply-to-ply interfaces. These layers were located in all the ply-to-ply interfaces of the glass fibre/epoxy laminates excluding the middle interface giving a total of 8 layers of nanofibers and epoxy resin added as compared to the virgin model. The elastic modules used are for a layer made of nylon nanofibers and epoxy resin. This is a $2 \mathrm{D}$ isotropic material as the nanofibers are randomly oriented in plane $\left(E_{2}=E_{3}\right)$. As the layer is thin, the properties are very different in the through thickness direction $\left(E_{1}\right)$. The material characteristics of uniform and continuous layers of nylon and epoxy resin are calculated according to the rule of mixture as indicated in [25-26]. The material constants are $\mathrm{E}_{1}=3 \mathrm{GPa}, \mathrm{E}_{2}=\mathrm{E}_{3}=0.75 \mathrm{GPa}, \mathrm{G}_{12}=\mathrm{G}_{13}=0.8 \mathrm{GPa}, \mathrm{G}_{23}=0.4 \mathrm{GPa}, \mathrm{v}_{12}=$ $0.35, v_{13}=v_{23}=0.2$ and $\rho=1140.0 \mathrm{Kg} \mathrm{m}^{-3}$.

The free vibratory response was excited applying a force of 10 Newtons in the red area at the negative $Y$ direction for 0.1 seconds (see Figure 4). This is used to simulate a short-time impulse force.

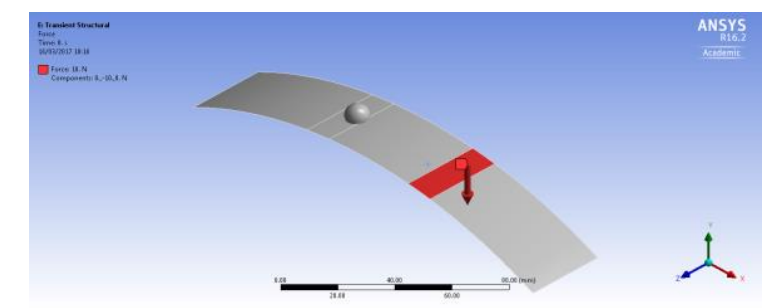

Figure 4: Excitation of the free decay response in composite beams.

\subsection{Finite element model precision and convergence}

The criteria employed to select the number of elements was the convergence [27]. When convergence is achieved, the values of the natural frequencies remain nearly constants. The element convergence study was done on the basis of the first five natural frequencies. Once 
a saturation is achieved (the frequencies do not change with the increase of the number of elements) it is considered that that method has converged.

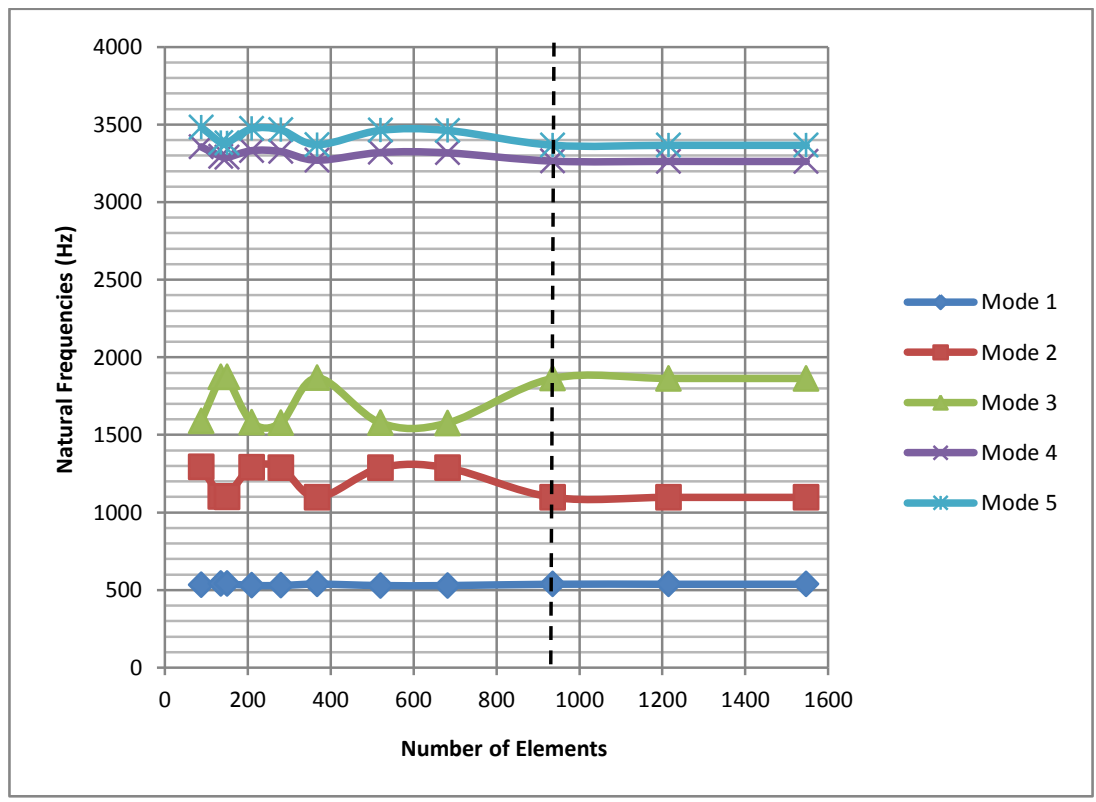

Figure 5: Effect of number of elements on the accuracy of natural frequency results

Figure 5 shows the first five natural frequencies for different generated number of elements. A considerable change in the results is obtained when increasing the number of elements from 88 to 936 . Meanwhile, the change in natural frequency obtained when increasing the number of elements from 936 to 1547 is negligible in all modes in consequence convergence is achieved. Figure 6 shows the percentage change in the natural frequencies with the number of elements. The results on Figure 6 also demonstrate that the change in the natural frequencies is not the same for the different modes. A considerable change is observed in the natural frequencies for Modes 2 and 3. Meanwhile, the change in the natural frequencies for Modes 1, 4 and 5 are quite small. These results show that some modes are more sensitive and fluctuate with the changing element size. In this particular case after 936 elements the changes in all frequencies were negligible (less than 0.05 . On the basis of these results it was decided to set the number of elements to 936 for all subsequent computations to achieve high accuracy for minimum computations time. Figure 7 shows the meshed sample with 936 elements. 


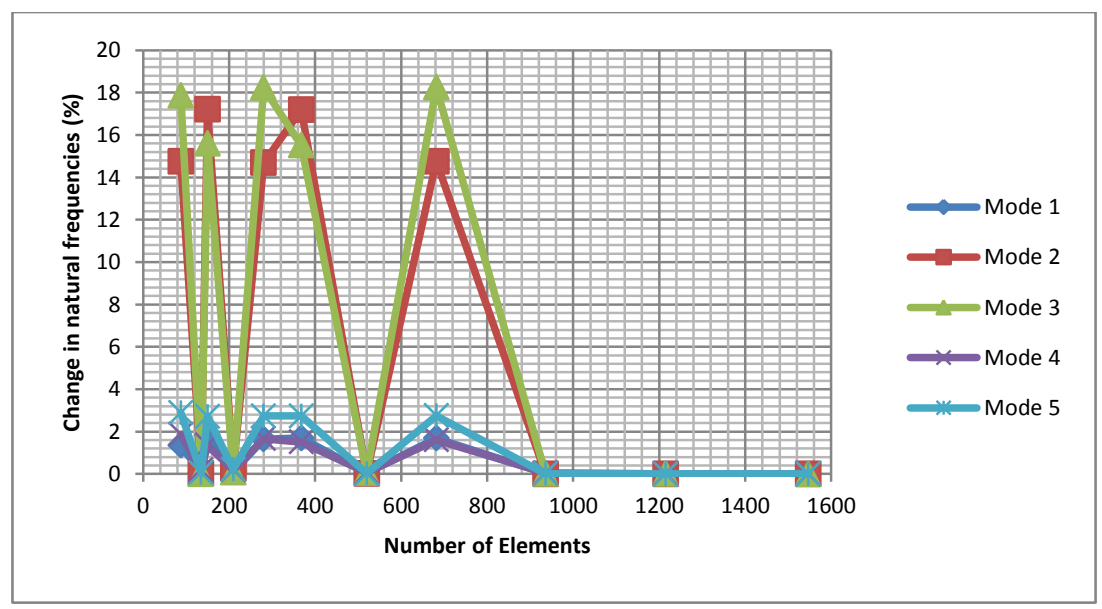

Figure 6: Effect of number of elements on the changes of natural frequency results

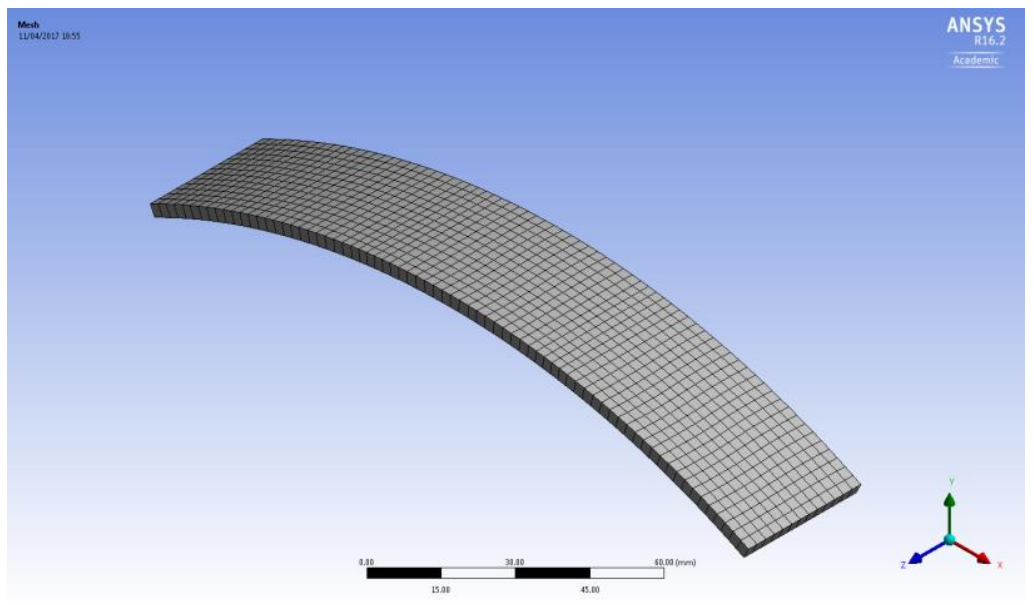

Figure 7 Image of the meshed sample with 936 elements

4.3. Comparison of FE model and experimental results: Transient vibration analysis versus modal analysis

The analysis of the vibrations in the finite element model was carried out by modal and transient vibration analysis. The results from the modal analysis did not agree with the experimental ones. However, the results from transient analysis were in line with experiments. So the natural frequencies of virgin and nano composites were calculated in FEM model in two different ways: assuming linear behaviour (modal) and non-linear behaviour (transient). The fundamental difference between linear and non-linear analysis is the stiffness. Linear analysis is based on the assumption that stiffness is constant. The force is linearly proportional to displacement therefore if the force doubles, the displacement is assumed to double in linear analysis. Non-linear analysis is based on the assumption that the stiffness is dependent on the displacement, so doubling the force does not necessary result in doubling the displacements. In general linear analysis provides an acceptable approximation to calculate the natural frequencies and damping ratio on most occasions however virgin and nano composites are characterized by a well-defined non-linear 
behaviour and linear analysis is not suitable to analyse the dynamical behaviour of these composites. Neglecting non-linear behaviour can lead to serious miscalculations in natural frequencies and damping ratio. Accordingly, linear analysis is not suggested as a simulation tool for the natural frequencies of the virgin and nano composites. Linear analysis is not appropriate for these types of composites as they demonstrate quite well defined nonlinear behaviour due to the multiple layers and the anisotropic structure.

To verify the results of the finite element model, the experimentally measured values of the natural frequency are compared with those obtained numerically, using linear (modal) and non-linear analysis (transient). Six samples were tested with the virgin polymer and 5 samples were tested made of nano-interleaved composite. Each experiment was repeated 10 times. The results given below are the average across the samples and for the 10 measurements. The standard deviation for the natural frequencies from the 10 measurements for the same sample are practically 0 , the values of the peaks did not change. The standard deviation across the samples is a bit bigger but it is still less than $2 \%$. So the experimental results in this case are rather stable and can be considered reliable/ credible. The errors provided below are actually differences/errors between the simulated values and the experimental ones. The Percentage Error (\%) defined in eq. (3) was used to estimate the error/difference of the model as compared to the experiments:

$$
\% \text { Error }=\operatorname{abs}\left(\frac{f_{\text {experimental }}-f_{\text {numerical }}}{f_{\text {numerical }}}\right) \times 100
$$

Where $f_{\text {experimental }}$ are the experimental values of the natural frequencies and $f_{\text {numerical }}$ are the natural frequencies calculated by ANSYS. Tables 1 and 2 show the natural frequencies obtained experimentally and numerically using linear analysis and the corresponding percentage errors. The average value of the percentage error in linear analysis was about $25 \%$. So it was concluded that the natural frequencies obtained numerically in the simulation do not have good agreement with the experimental ones. Accordingly, linear analysis is not suggested as a simulation tool for the natural frequencies of the virgin and nano composites. Linear analysis is not appropriate for these types of composites as they demonstrate quite well defined nonlinear behaviour due to multiple layers and anisotropic structure.

\begin{tabular}{|c|c|c|c|}
\hline Frequencies & Experimental & Linear & Percentage Error \\
\hline$f_{1}$ & 484.45 & 538.15 & 9.98 \\
\hline$f_{2}$ & 930.93 & 1098.20 & 15.23 \\
\hline$f_{3}$ & 1373.75 & 1866.90 & 26.42 \\
\hline$f_{4}$ & 1857.50 & 3270.20 & 43.20 \\
\hline$f_{5}$ & 2303.75 & 3370.20 & 31.64 \\
\hline
\end{tabular}

Table 1: Comparison between linear and experimental natural frequency results in $\mathrm{Hz}$ for virgin composite 


\begin{tabular}{|c|c|c|c|}
\hline Frequencies & Experimental & Linear & Percentage Error \\
\hline$f_{1}$ & 498.80 & 561.49 & 11.16 \\
\hline$f_{2}$ & 965.90 & 1107.30 & 12.77 \\
\hline$f_{3}$ & 1434.80 & 1898.70 & 24.43 \\
\hline$f_{4}$ & 1929.00 & 3322.00 & 41.93 \\
\hline$f_{5}$ & 2399.30 & 3377.50 & 28.96 \\
\hline
\end{tabular}

Table 2: Comparison between linear and experimental natural frequency results in $\mathrm{Hz}$ for nano composite

Tables 3 and 4 show the results for the first five natural frequencies obtained experimentally and numerically using nonlinear analysis. The average percentage error is about $4 \%$ when nonlinear analysis. So the results indicate that the natural frequencies obtained theoretically by nonlinear analysis match quite well with experimentally obtained ones.

\begin{tabular}{|c|c|c|c|}
\hline Frequencies & Experimental & Non Linear & Percentage Error \\
\hline$f_{1}$ & 484.45 & 515.00 & 5.93 \\
\hline$f_{2}$ & 930.93 & 940.00 & 0.96 \\
\hline$f_{3}$ & 1373.75 & 1330.00 & 3.29 \\
\hline$f_{4}$ & 1857.50 & 1770.00 & 4.94 \\
\hline$f_{5}$ & 2303.75 & 2420.00 & 4.80 \\
\hline
\end{tabular}

Table 3: Comparison between nonlinear and experimental natural frequency results in $\mathrm{Hz}$ for virgin composite

\begin{tabular}{|c|c|c|c|}
\hline Frequencies & Experimental & Non Linear & Percentage Error \\
\hline$f_{1}$ & 498.80 & 535.00 & 6.77 \\
\hline$f_{2}$ & 965.90 & 945.00 & 2.21 \\
\hline$f_{3}$ & 1434.80 & 1343.00 & 6.84 \\
\hline$f_{4}$ & 1929.00 & 1775.00 & 8.98 \\
\hline$f_{5}$ & 2399.30 & 2423.00 & 0.98 \\
\hline
\end{tabular}

Table 4: Comparison between nonlinear and experimental natural frequency results in $\mathrm{Hz}$ for nano composite

It can be seen from the above tables that transient analysis gives a much better agreement with the experimentally measured natural frequencies. Accordingly transient analysis is suggested in this study to simulate the free vibratory behaviour of the virgin and nano interleaved composites. The following figures 8 and 9, also demonstrate that, nonlinear analysis has a better agreement with experimental data than linear analysis. The graphs show that the percentage error (\%E) in nonlinear analysis is clearly smaller than the percentage error $(\% \mathrm{E})$ in linear analysis. These differences are again due to the nature of the analysis; linear analysis assumed a linear behaviour and nonlinear analysis assumed a nonlinear behaviour. 


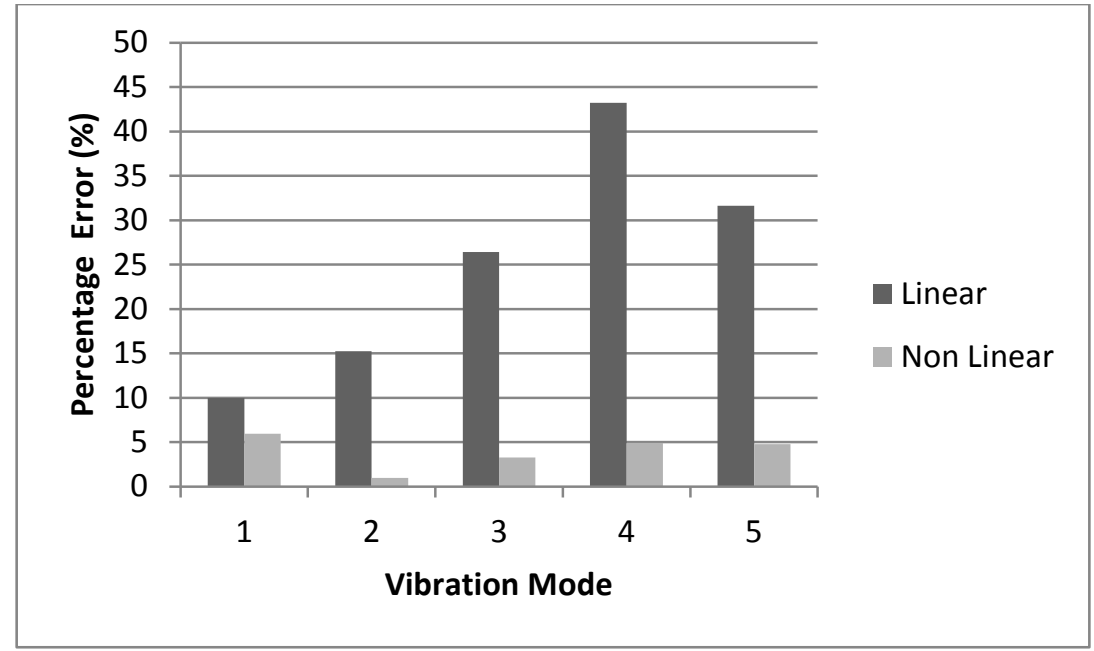

Figure 8: Comparison of percentage error for linear and non-linear analysis in virgin composite

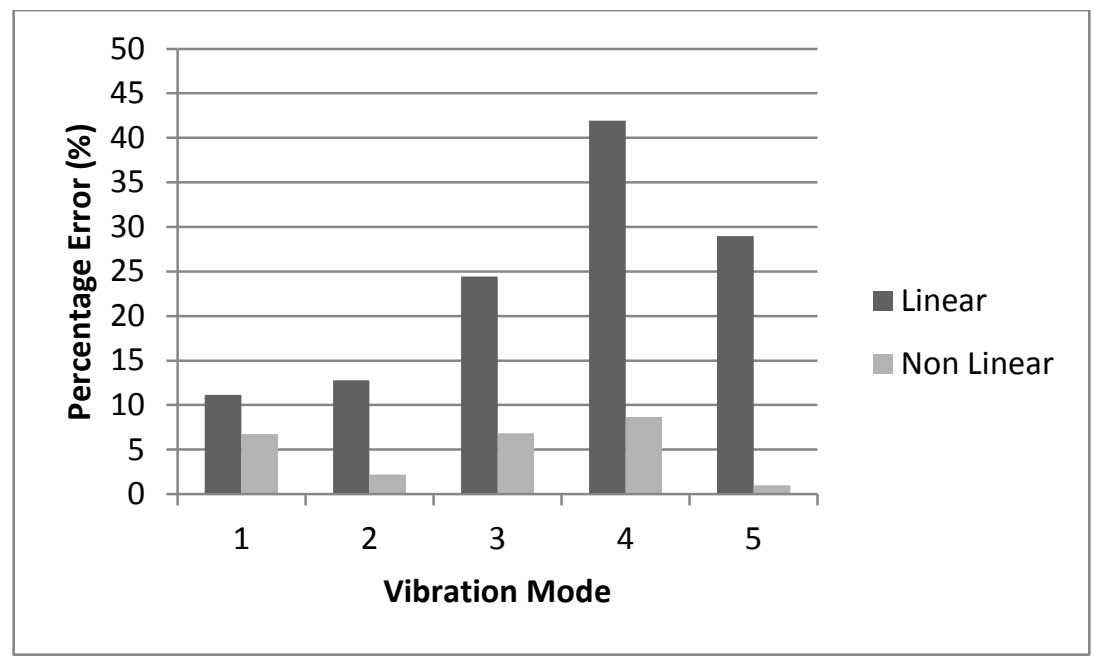

Figure 9: Comparison of percentage error for linear and non-linear analysis in nano composite

\section{Effect of Nylon nano-fibres on the macro-dynamic properties of GFRP}

\subsection{Effect of nanofibers on the natural frequencies}

In this paragraph the effect of nano-fibres on the natural frequencies is studied. For the purpose glass fibre epoxy beams which are clamped-clamped at both ends are analysed. To address this problem, the natural frequencies of the beams made of virgin composites are compared to the natural frequencies of the beams made of nano enriched composites. The effect of nanofibers in composites is analysed through the variation of the natural frequencies for the beams made of virgin and the nano-enriched composites. The percentage difference between the experimental and the numerically obtained natural frequencies is used for the purpose (see equation (4)). 


$$
\% \text { Difference }=\left(\frac{f_{\text {nano }}-f_{\text {virgin }}}{f_{\text {virgin }}}\right) \times 100
$$

Where $f_{\text {nano }}$ are the natural frequencies of the nano enriched beams and $f_{\text {virgin }}$ are the natural frequencies of the virgin beams. The experimental results given in Table 5 shown the first five modes of vibration calculated experimentally by free decay vibration tests in virgin and nano composites. These results indicate that the presence of nano-fibres in the composites increases the natural frequencies with up to $4.25 \%$.

\begin{tabular}{|c|c|c|c|}
\hline Frequencies $(\mathrm{Hz})$ & Virgin & Nano & $\begin{array}{c}\text { Variation of Natural } \\
\text { Frequency (\%) }\end{array}$ \\
\hline $\mathrm{f}_{1}$ & 484.45 & 498.80 & 2.88 \\
\hline $\mathrm{f}_{2}$ & 930.93 & 965.90 & 3.62 \\
\hline $\mathrm{f}_{3}$ & 1373.75 & 1434.80 & 4.25 \\
\hline $\mathrm{f}_{4}$ & 1857.50 & 1929.00 & 3.71 \\
\hline $\mathrm{f}_{5}$ & 2303.75 & 2399.30 & 3.98 \\
\hline
\end{tabular}

Table 5: Variation of natural frequencies due to nanofibers calculated experimentally

Table 6 shows the values of natural frequencies calculated numerically using no-linear transient analysis. It can be seen that according to these results the increase in the natural frequencies due to nanofibers is very small for the five modes of vibration (variation of natural frequency less than 3.74). These numerical results show that the inclusion of nylon nanofibers has a small impact on the natural frequencies.

\begin{tabular}{|c|c|c|c|}
\hline Frequencies $(\mathrm{Hz})$ & Virgin & Nano & $\begin{array}{c}\text { Variation of Natural } \\
\text { Frequency (\%) }\end{array}$ \\
\hline $\mathrm{f}_{1}$ & 515.00 & 535.00 & 3.74 \\
\hline $\mathrm{f}_{2}$ & 940.00 & 945.00 & 0.53 \\
\hline $\mathrm{f}_{3}$ & 1330.00 & 1343.00 & 0.97 \\
\hline $\mathrm{f}_{4}$ & 1770.00 & 1775.00 & 0.28 \\
\hline $\mathrm{f}_{5}$ & 2420.00 & 2423.00 & 0.12 \\
\hline
\end{tabular}

Table 6: Variation of natural frequencies due to nanofibers calculated theoretically by nonlinear analysis

It can be seen that the natural frequencies show a slight increase as a result of the inclusion of nanofibers into the ply-to-ply interfaces. This is according to the experimental as well as the simulation results, although the simulation results actually show negligible increase in all the frequencies except the first one. The increase in the natural frequencies is evident from the experimental measurements and also from the numerical calculations. But in general the increase in all the first 5 natural frequencies is rather small and thus inconclusive as these changes are within the region of the precision of the experiments. The numerical results as mentioned show no change for all the frequencies except the first one. Thus according to our results the natural frequencies do not appear to change as a result of the nano-enrichment. 
Shiuh-Chuan Her et al [28] analysed the natural frequencies on composites reinforced with carbon nanotubes. This work showed that natural frequencies increased due to the addition of carbon nanotubes with up to $7 \%$ as compared to the virgin composite. The same paper also indicated that the increase of natural frequencies depends on the content of carbon nanotubes. G. Balaganesan et al [4] analysed the natural frequencies on virgin and clay nano-enriched composites. The results showed that natural frequencies increased with up to $12 \%$ for the nano-enriched material. These authors of [4] also concluded that the increase of the natural frequencies depends on the content of clay nanofibers. This work demonstrated that nano-enriched composites showed a considerable increase in the natural frequencies as compared to the virgin panels when the content of clay nanofibers increased up to $3 \%$. However, a marginal increase in the natural frequencies was observed for more than $3 \%$ contents of clay nanofibers. The possible reason of the marginal increase when the content of clay nanofibers is more than 3\% might be that the formation of clusters of nanofibers. Others authors as $[29,30]$ also observed a very slight change in the natural frequencies due to inclusion of nanofibers. In general the change in the stiffness of the nano-enriched composite depends on nano-inclusion and its properties.

In this particular case, the natural frequencies do not change as a result of the nanoenrichment with nylon. The changes on the natural frequencies are negligible (less than $5 \%$ ). The natural frequencies are directly proportional to the stiffness and inversely proportional to the mass. The stiffness of nylon nanofibers is much smaller than the stiffness of glass fibers thus it is expected that nylon nanofibers will not improve the overall stiffness. The changes in mass and stiffness due to the nano-enrichment with nylon nanofibers are minuscule. Consequently, the small/ negligible change in the natural frequencies is expected as the nylon enrichment cannot contribute in terms of stiffness. So it can be concluded that the natural frequencies of nano modified composites will be very much the same as the natural frequencies of the virgin composites.

In any case from this research it can be concluded that the nylon nano interleaving can preserve the frequency range of a structure for the case of free and forced vibration and thus the modified structure will be expected to vibrate within the same frequency range and have approximately the same resonances. So the nano enriched structure can be designed at least in terms of frequencies as the original structure made of the virgin composites.

\subsection{Effect of nanofibers on the damping ratio}

In this section, the effect of nanofibers on the damping ratio of glass fibres epoxy beams is analysed. This was carried out by comparing the damping ratio of the virgin and the nanoenriched composite beams. Their percentage difference as defined by equation (5) is used for the purpose: 


$$
\% \text { Difference }=\left(\frac{\zeta_{\text {nano }}-\zeta_{\text {virgin }}}{\zeta_{\text {virgin }}}\right) \times 100
$$

Where $\zeta_{\text {virgin }}$ is the damping ratio of the beam made of virgin composite and $\zeta_{\text {nano }}$ is the damping ratio of the beam made of the nano-enriched composite. The results given in Table 7 show the damping ratio calculated experimentally and numerically using ANSYS nonlinear analysis in virgin and nano composites. The damping ratio was calculated using the logarithmic decrement method explained in section 3 . The experimental and the numerical results show that the enrichment of composites with nylon nanofibers increased the damping ratio with a $36.36 \%$ and $45.63 \%$ respectively.

Overall the results calculated numerically agree with the experimental observations in the sense that both results show a significant increase in the damping. The values are not the same and they differ with about $20 \%$. This will be investigated further in our future work. On the basis of the above results it can be concluded qualitatively that the inclusion of nanofibers increases considerably the damping ratio.

\begin{tabular}{|c|c|c|c|}
\hline $\begin{array}{c}\text { Damping Ratio } \\
\text { (dimensionless) }\end{array}$ & Virgin & Nano & $\begin{array}{c}\text { Variation of Damping Ratio } \\
(\%)\end{array}$ \\
\hline Experimentally & 0.0121 & 0.0165 & 36.36 \\
\hline Nonlinear Analysis & 0.0160 & 0.0233 & 45.63 \\
\hline
\end{tabular}

Table 7: Variation in damping ratio due to nanofibers calculated experimentally and numerically

The damping ratio is a measure of the amount of energy which a material can dissipate under an impact. The higher the damping ratio, the more energy is dissipated. This is beneficial as high damping materials can be used to reduce the vibration amplitudes in structures and also to alleviate the effect of resonances. Table 7 shows the percentage change in the damping ratio as obtained experimentally and by the Ansys nonlinear analysis. In both cases the significant increase is evident. Our experimental results show $37 \%$ increase for the case of the numerically simulated response the increase is $45 \%$. The trend in both responses- the experimentally measured and the numerically simulated ones- is the same. There is a difference of about $7 \%$ between the two cases which can be attributed to the divergence of the numerical model from the real case and also to measurement and computational errors.

Ultimately on the basis of both results it can be deducted that the inclusion of nanofibers into the ply-to-ply interfaces increases the damping considerably. The increase of the damping ratio can be explained by the fact that nano-enriched composites are characterized by a larger interface area between fibres, nanofibers and matrix as compared to the virgin composites. The increase in the interface area is expected to increase the energy which is dissipated. 
Other authors found similar results for nylon nano enriched composites: A similar effect was observed in [17] which investigated the impact resistance under low velocity impact of nylon nanofibrous modified composite laminates. This work found that damping ratio of carbon fibre composite panels reinforced with nylon nanofibers is about $62 \%$ higher than the damping of virgin panels. Another study conducted by Jihua Gou et al [29] indicated that the damping ratio of composite laminates enriched with carbon nanotubes was up to $200 \%$ higher as compared to the damping in virgin composites. Naser Kordani et al [30] also concluded that the damping ratio can increase considerably due the inclusion of nanofibers. This paper showed experimentally that the inclusion of carbon nanotubes in composites increased considerably the damping ratio. K.T.B Padal et al [18] showed that the damping ratio of epoxy composites reinforced with jute nanofibers increased up to $70 \%$ as compared to the damping ratio of the virgin panels. In conclusion it can be said that the enrichment with nano-fibres causes a significant increase in the damping. The individual effect depends of course on the particular nano material used, but so far all studies report a significant increase in the damping ratio as a result of nano-inclusions. As previously mentioned this can be used to the benefit of structures in order to reduce the amplitude of the experienced vibration. Thus nano-enrichment can be used to develop structures with higher damping which will experience much smaller vibration amplitudes. This effect can be beneficial in the development of a great variety of structures including civil, mechanical and aerospace. Reduction of vibrations is required in a number of applications including such from our everyday life experience like e.g vehicles, bridges, buildings. The higher damping can also increase the impact resistance of the nano modified material as more energy will be dissipated during the impact. This again can have a beneficial effect for a number of structures in different applications.

\subsection{Effect of nanofibers on the stiffness}

Paragraph 5.1 studied the effect of nano-inclusions on the natural frequencies and it was found that they do not have a significant effect on the natural frequencies. To further analyse this effect, the influence of nanofibers on the stiffness was investigated. The experiment was done by comparing the stiffness of virgin and nano-enriched composites. The structures tested numerically are a beam made of virgin composites and other beam made of nano-enriched composites. The effect of nanofibers was studied through the percentage variation of the stiffness of virgin and nano-enriched composites using equation (6):

$$
\% \text { Difference }=\left(\frac{k_{\text {nano }}-k_{\text {virgin }}}{k_{\text {virgin }}}\right) \times 100
$$

Where $k_{\text {nano }}$ is the stiffness of the beam made of nano-enriched composite and $k_{\text {virgin }}$ is the stiffness of the beam made of virgin composite. Table 8 shows the stiffness in $\mathrm{N} / \mathrm{mm}$ 
calculated theoretically by Hooke's law for the virgin and the nylon nano interleaved composites. The results show that the inclusion of nylon nanofibers into the composites did not affect the stiffness. Thus in this particular case one cannot expect a stiffness increase as the stiffness of the nano-included material is much lower than the stiffness of the main composite. In general nano-inclusions may affect significantly a certain characteristic/property which is high/well expressed for the material used as nano-inclusion. Like e.g. carbon nano-inclusions can affect the conductivity and e.g. rigid silica nano particles can increase the stiffness of the matrix system. On a number of occasions nano inclusions and composites in general aim to achieve higher stiffness. In [31] the authors found experimentally that the effect of aligned carbon nanotube sheet interlayers in woven glass fibre composites increases the stiffness. Stiffness of nylon nanofibers is actually rather low so it cannot be used for purposes of making the material more rigid. Thus the very low/no change in the natural frequencies can be explained in this case by the fact that the stiffness of the nano-modified composite is practically not affected by the nanofibers of nylon.

\begin{tabular}{|c|c|c|c|}
\hline Stiffness (N/mm) & Virgin & Nano & Variation of Stiffness (\%) \\
\hline Non-linear analysis & 427.4 & 427.6 & 0.1 \\
\hline \multicolumn{2}{|c|}{ Table 8: Variation in stiffness due to nanofibers calculated numerically }
\end{tabular}

Table 8: Variation in stiffness due to nanofibers calculated numerically

The small (within the error range) change of the natural frequencies is mainly due to the lack of stiffness increase. Thus the combination of very low/no stiffness and mass change should be expected to result in either very low/no frequency change. This can be also used to its benefit as the structure made of the nano modified material will preserve the working frequency range of the structure made of the nano-material while in the same time increasing the damping.

\section{General Conclusions and Discussion}

The main aim and the main achievement of this study is the analysis of the effect of nylon nano-layers on some dynamic properties of the nano-enriched GFRP composite. This was done experimentally and also using a relatively simple FE element model. The results from the modelling and the experiments are in good agreement. Thus the authors succeeded to demonstrate that for the purposes of dynamic modelling a transient FE dynamic model is able to give adequate simulation of the dynamic properties and behaviour of the nanomodified structure. The authors are on the opinion that such a model is rather flexible and its simplicity makes it easy to use for other applications and for the case of predicting the dynamic behaviour of other nano-enriched materials. 
The study investigates primarily the effect of nylon nano-layers on the macro-dynamic characteristics, namely the natural frequencies and the damping, of the resultant material. These characteristics are rather important for the purposes of designing structures as most structures vibrate while being in use. Our research found that the natural frequencies of the nano-modified structure are not affected (the effect is within the range of the experimental/ measurement error) by the nylon nanofiber, while its damping is significantly affected. The damping goes up with nearly $40 \%$ according to our experimental results which is confirmed by the numerical investigation. This can be explained by the increase in the interface area between the fibres, the nano fibres and the matrix, which is expected to result in higher energy dissipation. Searching to further confirm/explain the effect of nylon nano fibres the authors investigated numerically the stiffness of the nano enriched polymers as compared to the virgin ones. According to our numerical results the stiffness undergoes very low/no change. This supports the finding. The negligible stiffness change combined with the low/no effect on the mass of the specimen supports the conclusion for the low frequency change of the natural frequencies of the nano-modified structure as compared to the virgin one.

The small change in the natural/resonant frequencies of the nano-modified structure combined with the damping increase can be used for designing structures from the nanomodified material for dynamic/vibratory purposes which will retain the working frequency range of the original structure (made of the virgin composite) while increasing the damping. This can be beneficial for purposes of reducing the vibration amplitude and also for alleviating the effect of resonance.

In conclusion it should be noted that this study contributes to the knowledge about the effect of nylon nanofibers on the global dynamic behaviour of GFRP composites in two aspects. It suggests a simple FE modelling procedure which is able to simulate the dynamic behaviour of the nano-enriched composites with a good accuracy for mast applications. It also establishes some general trends in the behaviour of macro dynamic properties of nylon interleaved GFRP composites as compared to the virgin (non-modified) structure. These can be used to improve the dynamic/vibratory properties of GFRP composites by interleaving them with nylon nano fibers.

\section{References}

1. A. Fereidoon, F. Memarian, Z. Ehsani. Effect of CNT on the Delamination Resistance of Composites. Journal of Fullerenes Nanotubes and Carbon Nanostructures 2013; 21(8):712-724.

2. H. Saghafi, R. Palazzetti, A. Zucchelli, G. Minak. Influence of electrospun nanofibers on the interlaminar properties of unidirectional epoxy resin/glass fiber composite laminates. Journal of Reinforced Plastics and Composites 2015; 34(11):907-914. 
3. K. Molnár, E. Košt'áková, L. Mészáros. The effect of needleless electrospun nanofibrous interleaves on mechanical properties of carbon fabrics/epoxy laminates. Journal of Express Polymer Letters 2013; 8(1):62-72.

4. G. Balaganesan, R. Velmurugan. Vibration and Energy Dissipation of Nanocomposite Laminates for Below Ballistic Impact Loading. Latin American Journal of Solids and Structures 2015; 12:2259-2280.

5. R. Palazzetti. Flexural behaviour of carbon and glass fiber composite laminates reinforced with Nylon 6,6 electrospun nanofibers. Journal of Composite Materials 2014; 49(27):3407-3413.

6. Bryan M. Tyson, S. M. Asce, Rashid K. Abu Al-Rub, Z. Grasley. Carbon Nanotubes and Carbon Nanofibers for Enhancing the Mechanical Properties of Nanocomposite Cementitious Materials. Journal of Materials in Civil Engineering 2015; 23(7):1028-1035.

7. D. Olmos, G. González-Gaitano, A. L. Kholkin, J. González-Benito. Flexible PVDF-BaTiO Nanocomposites as Potential Materials for Pressure Sensors. Journal of Ferroelectrics 2013; 447(1):9-18.

8. Alamusi, N. Hu, H. Fukunaga, S. Atobe, Y. Liu, J. Li. Piezoresistive Strains Sensors Made from Carbon Nanotubes Based Polymer Nanocomposites. Journal of Sensors 2011; 11:10691-10723.

9. D. Garcia, I. Trendafilova, Daniel J. Inman. A study on the vibration-based selfmonotoring capabilities of nano-enriched composite laminated beams. Journal of Smart Materials and Structures 2016; 25(4):1-13.

10. Min Wook Lee, Seongpil An, Hong Seok Jo, Sam S. Yoon, Alexander L. Yarin. Self-Healing Nanofiber-Reinforced Polymer Composites. 1. Tensile Testing and Recovery of Mechanical Properties. Journal of Applied Materials \& Interfaces 2015; 7(35):1954619554.

11. Lee MW, Yoon SS, Yarin AL. Solution-Blown Core-Shell Self-Healing Nano- and Microfibers. Journal of Applied Materials \& Interfaces 2016; 8(7):4955-62.

Lee MW, An S, Lee C, Liou M, Yarin AL, Yoon SS. Hybrid self-healing matrix using coreshell nanofibers and capsuleless microdroplets. Journal of Applied Materials \& Interfaces 2014; 6(13):10461-8.

P. Ribeiro, O. Thomas. Nonlinear Modes of Vibration and Internal Resonances in Nonlocal Beams. Journal of Computational and Nonlinear Dynamics 2017; 12:1-11. 
14. A. Fereidoon, R. Rafiee and R. Maleki Moghadam. Modal analysis of carbon nanotube reinforced polymer using multi-scale finite element method. Journal Mechanics of Composite Materials 2013. 49(3): 325-332.

15. R. Rafiee and R. Maleki Moghadam. Simulation of impact and post-impact behavior of carbon nanotube reinforced polymer using multi-scale finite element modelling. Journal of Computational Materials Science 2012. 63:261-268.

D. C. Prevorsek, Y. D. Kwon, and R. K. Sharma. Interpretive Nonlinear Viscoelasticity: Dynamic Properties of Nylon 6, Nylon 66, and Nylon 12 Fibers. Journal of Applied Polymer Science 1980. 25:2063-2104.

R.Palazzetti, A.Zucchelli and I.Trendafilova. The self-reinforcing effect of Nylon 6,6 nanofibres on CFRP laminates subjected to low velocity impact. Journal of Composite structures 2013. 106: 661-671

K.T.B Padal, K. Ramji, VVS Prasad. Damping Behaviour of Jute Nano Fibre Reinforced Composites. International Journal of Emerging Technology and Advanced Engineering 2014; 4(4):753-759.

D. Garcia, R. Palazzetti, I. Trendafilova, C. Fiorioni, A. Zucchelli. Vibration-based delamination diagnosis and modelling for Composite laminates plates. Journal of Composite Structures 2015; 130:155-162. D. Di Maio, Ad. Carri, F. Magi, I. A. Sever. Detection of nonlinear behaviour of composite components before and after endurance trials. Journal of Nonlinear Dynamics 2014; 2:83-95.

M. Hammami, A. El Mahi, C. Karra, M. Haddar. Nonlinear behaviour of glass fibre reinforced composites with delamination. Journal of Composites 2016; 92:350-359.

L.B. Magalas and T. Malinowski. Measurement Techniques of the Logarithmic Decrement. Journal of Solid State Phenomena 2003. 89: 247-260.

H. Mevada and Dipal Patel. Experimental determination of structural damping of different materials. 12 ${ }^{\text {th }}$ International Conference on Vibration Problems, ICOVP 2015.

Hamed Saghafi. Mechanical behaviour of flat and curved laminates interleaved by electrospun nanofibers. PhD Thesis University of Bologna 2013.

25. P. Zhu, Z.X. Lei, K.M. Liew. Static and free vibration analyses of carbon nanotubereinforced composite plates using finite element method with first order shear deformation plate theory. Journal of Composite Structures 2012. 94:1450-1460. 
26. M.H. Yas, N. Samadi. Free vibrations and buckling analysis of carbon nanotubereinforced composite Timoshenko beams on elastic foundation. International Journal of Pressure Vessels and Piping 2012. 98: 119-128.

27. K. Alnefaie. Finite element modelling of composite plates with internal delamination. Journal of Composite Structures 2009; 90:21-27.

28. Shiuh-Chuan Her, Chun-Yu Lai. Dynamic Behaviour of Nanocomposites Reinforced with Multi-Walled Carbon Nanotubes (MWCNTs). Journal of Materials 2013; 6(6):2274-2284.

29. J. Gou, S. O'Braint, H. Gu, G. Song. Damping Augmentation of Nanocomposites Using Carbon Nanofiber Paper. Journal of Nanomaterials 2006; 2006:1-7.

30. N. Kordani, A. Fereidoon, M. Ashoori. Damping Augmentation of Nanocomposites Using Carbon Nanotube/Epoxy. Journal of Structural Dynamics 2011; 3:1605-1615. Nanotube Sheet Interlayers. Journal of Nanomaterials 2015; 1-9. 